\title{
The Effects of Density Difference on Displacement Interface in Eccentric Annulus During Horizontal Well Cementing
}

\author{
Feng Fuping*, Ai Chi, Yu Fahao, Cui Zhihua, Fan Sen and Xu Haisu \\ Key Laboratory of Education Ministry for Enhanced Oil Recovery, Northeast Petroleum University, Daqing 163318, \\ China
}

\begin{abstract}
It is known that the interface of slurry displacement during cementing reflects the extent of two-phase fluid intermixing. A longer interface means that more displacing fluid becomes contaminated. When the interface becomes too long, it can occur that the trailing edge of the interface fails to reach a designed cementing segment at the end of operation, which reduces displacement effectiveness and adversely affects cementing quality and well integrity. In this paper, a 3D numerical model was developed and employed to analyze the effects of slurry density difference on the displacement interface in the eccentric annulus of horizontal wells. The simulation results showed that smaller density difference can create shorter interface and lead to better displacement efficiency when casing is centrally placed in a horizontal borehole. When the casing is eccentric, slurry tends to advance in the annular upper side under smaller density difference; otherwise, the opposite result will be obtained under greater density difference. Therefore, there exists an optimal density difference under which a minimum interface length can be achieved for a given casing eccentricity. In addition, the optimal density difference increases as the shear thinning index, consistency index and yield point of a cement slurry increase. In order to minimize the intermixing extent of two-phase fluids and slurry fingering effects in cementing operations, it is necessary to take into consideration the comprehensive influence of casing eccentricity, slurry density, and rheological parameters to design slurry parameters properly, which will achieve higher displacement efficiency and better cementing quality.
\end{abstract}

Keywords: Horizontal wells, eccentric annulus, displacement interface, density difference, slurry fingering.

\section{INTRODUCTION}

Slurry displacement interface reflects the intermixing extent of two-phase fluids during cementing. When the mixing interface becomes too long, it can occur that the trailing edge of the interface fails to reach a designed cementing segment at the end of operation, which reduces displacement effectiveness and adversely affects cementing quality and well integrity. There is no doubt that a bad annular cementing quality can lead to any of the following problems or disasters: blowout, leakage at surface, destruction of subsurface ecology, potential contamination of freshwater, delayed or failed abandonment, as well as loss of revenue. Many factors can affect the displacement interface shape [1-9], such as:

\subsection{Fluids' Properties}

Fluids used in cement includes drilling fluid, spacer and slurry. Reducing the drilling fluid's gel strength, yield stress, and plastic viscosity is recognized as being very beneficial, because the driving force necessary to displace the mud are reduced, and its mobility is increased. In order to achieve good cementing effect, The yield point and plastic viscosity ratio should satisfy the relationship, drilling mud's $<$ spacer's $<$ slurry's, under which the velocity profile will advances level by level.

*Address correspondence to this author at the No.199 FaZhan Road, HighTech Development Zone, Daqing 163318, China; Tel: 13904696932;

Fax: 0459-6503482; E-mail: fengfuping2005@163.com

\subsection{Wellbore Asperity and Annular Size}

Bigger asperity can generate greater resistance for fluids on the wellbore wall, which decreases fluids' velocity with no doubt. However, it has no impact on the velocity at annular centerline. As a result, the displacement interface length becomes longer. Increasing annular clearance can decrease the flow resistance, which benefits for achieving a stale interface. With an optimal range of sheath thickness of 1.5 inches with proper centralization or standoff requirements of a minimum of $70 \%$. For a particular well in question, the optimum values for these parameters should, however be calculated from programs that consider cement slurry placement and cement sheath integrity.

\subsection{Casing Centralization/Standoff}

It is well known that if casing is more center in vertical well, the flow resistance difference between annular wide and narrow clearance will be smaller, which leads to a more uniform flow for fluids and a shorter interface. So casing centralization will achieve the best cementing quality for vertical well. However, when casing is center in horizontal well, the displacement interface will advances along the wellbore low side because of the driving force caused by density difference. An appropriate casing's eccentricity can generate flow resistance for advancing slurry, which decrease its velocity. Finally, the interface becomes shorter. Therefore, for displacement with density difference in horizontal well, good cementing quality may not be achieved 
when casing is center. We will discuss the phenomenon explicitly in the context.

\subsection{Casing Movement (Reciprocation and/or Rotation)}

The casing movement either rotation or reciprocation cannot only make drilling fluid and slurry in a sheared state but also form a backflow on the outside of annular wide clearance. The two effects can break drilling fluid's gel structure and accelerate the retained drilling fluid in the annular narrow clearance to flow.

\subsection{Flow Rates}

Flow rates achieved during the circulation stage before and during the cementing and displacement stage have a significant effect on mud displacement. The turbulent flow is the most beneficial flow regime for improving displacement efficiencies, followed by the plug flow, and the laminar flow is worst. In actual cementing operation, being turbulent flow for viscous slurry in annular clearance need high injecting capacity and pressure, unfortunately, which are always limited by pump power and wellbore configuration. So the turbulent flow can't be achieved easily. For plug flow displacement, it always limited by slurry's thickening time. In most situations, laminar flow displacement is common. However, because the velocity profile of laminar flow is leptokurtic, which results in slurry advancing in the center, interface can't maintain stable in cement. In order to guarantee the velocity profile to advance level by level and decrease interface length, turbulent spacer displacement at lowreturn velocity is recommended to use.

\subsection{Density Difference Between Displacing and Dis- placed Fluid}

Driving forces, produced by a density difference between the two fluids, influence the breakdown of gel structure of the drilling fluid and, therefore, may enhance the stability of displacement. For vertical well, greater density difference can obtain good displacement effect when meeting anti-leak condition. For slim well and horizontal well, increasing density difference will result in slurry advancing on the annular low side and slurry advancing happens on the upper annular side under smaller density difference. Therefore, there exists an appropriate density difference to make the interface length minimum for a certain casing's eccentricity, which is views expressed in the context.

Density difference is the key factor to affect displacement interface stability. Many scholars have concluded that increasing density difference is beneficial for enhancing displacement effect in vertical well by the method of theoretical studies and laboratory experiments. However, for slim and horizontal well, the conclusion may not be correct considering complex loading conditions. The reasons are as follow:

1) The casing eccentricity. Casing tends to move toward the low side of a wellbore due to gravity, which creates a narrow annular clearance. Because of greater resistance for fluid flow through the narrow clearance, it is easy for cement slurry to advance through a wide clearance on the upper side of the wellbore. As a result, the interface will become longer and more complex.
2) The density difference between cement slurry and drilling mud. Heavy slurry tends to flow toward the lower side in the wellbore and light drilling mud always gathers in the upper side, leading to the advancement of cement slurry in the upper side of the wellbore and drilling-mud retention in the lower side of the wellbore. Therefore, a non-density-difference cementing technique is advised to use in the horizontal well when the casing is central [10, 11].

The position and extent of displacement interface fingering depend on the relative relationship between resistance caused by casing eccentricity and buoyancy caused by density difference. If the density difference is greater, the buoyancy will be more than the resistance. As a result, the slurry advances in the lower side of the wellbore. However, if the density difference is smaller, the opposite results will be obtained. Therefore, either too large or too small in density difference will give rise to the instability of displacement interface for cementing eccentric annulus of horizontal wells. It is obvious that greater density difference may not result in better cementing quality, which is significantly different from the vertical well. There exists an optimum density difference for a given casing eccentricity, and the created buoyancy can balance out the resistance in annular clearance. As a result, a stable displacement interface and optimum displacement efficiency are achieved in cementing. In recent studies, a few scholars have analyzed this phenomenon, but they did not successfully find the matching relationship between casing eccentricity and density differences in horizontal well cementing. As a result, they could not provide an effective design scheme of displacing fluids in the eccentric annulus of horizontal wells [12-14].

The comprehensive influence of resistance and buoyancy effects on the interface is studied in the paper. The displacement interface shape under various eccentricity, density difference and rheological parameters has been simulated and analyzed using the method of 3D numerical simulation. In order to enhance the stability of displacement interface, reduce the intermixing extent of two-phase fluids and minimize the interface length, a realistic matching relationship between casing eccentricity and density difference in the horizontal well has been obtained in this study. In addition, the effects of shear thinning index, consistency index and yield point of the cement slurry on the optimal density difference with respect to various casing eccentricity values has been analyzed.

\section{GOVERNING EQUATIONS AND SOLUTIONS}

\subsection{Governing Equations}

The flow characteristics of slurry and drilling fluid during cementing can be accurately described by the basic equations of fluid mechanics, including the momentum conservation equation, the continuity equation and the volume fraction equation. These equations constitute the governing equations for numerical simulation.

\subsubsection{Momentum Conservation Equations}

The momentum conservation equations are 3-D unsteady Navier-Stokes equations, which are given by: 


$$
\begin{aligned}
& \frac{\partial(\rho u)}{\partial t}+\operatorname{div}(\rho u \boldsymbol{U})=\operatorname{div}(\mu \operatorname{grad} u)-\frac{\partial p}{\partial x}+F_{x} \\
& \frac{\partial(\rho v)}{\partial t}+\operatorname{div}(\rho v \boldsymbol{U})=\operatorname{div}(\mu \operatorname{grad} v)-\frac{\partial p}{\partial y}+F_{y} \\
& \frac{\partial(\rho w)}{\partial t}+\operatorname{div}(\rho w \boldsymbol{U})=\operatorname{div}(\mu \operatorname{grad} w)-\frac{\partial p}{\partial z}+F_{z}
\end{aligned}
$$

where $u, v$ and $w$ represent the velocities in $x, y$, and $z$ direction, respectively. $\mathrm{P}$ is pressure. $F_{\mathrm{x}}, F_{\mathrm{y}}$ and $F_{\mathrm{z}}$ are the volume forces in the $x, y$ and $z$ directions respectively. $\rho$ is the density of the fluid and $\mu$ is the viscosity.

\subsubsection{Continuity Equation}

The continuity equation is given by:

$$
\frac{\partial \rho}{\partial t}+\operatorname{div}(\rho \boldsymbol{U})=0
$$

\subsubsection{Volume Fraction Equation}

The fluid flow is assumed through an annular space between the rock formation, and the annular space is initially filled with the drilling fluid before simulation. When the simulation starts, the slurry is injected at a constant flow rate in the central tube. The volume of fluid (VOF) method is used to take into account the multiphase flow. The VOF method is used to solve a set of mass conservation equations and to obtain the volume fraction $\alpha_{i}$ of each phase, which should sum up to unity inside each control volume. $f^{i}$ is defined as the volume function that elaborates the volume of $i$ fluid in a control body. $f^{i}(r, \theta, z)=0$ means that there is no $i$ fluid at point $(r, \theta, z)$ and $f^{i}(r, \theta, z)=1$ indicates that there exists only $i$. When simulating in the Fluent software, the volume function is solved in every position of one grid unit based on given boundary conditions and the initial values. The mean value derived from integration of volume function in the grid unit is defined as the volume fraction $\alpha_{\mathrm{i}}$ for fluid $i$. If the unit only contains $i$ fluid, its volume fraction $\alpha_{\mathrm{i}}$ is 1 ; otherwise, the volume fraction is 0 . If there exists an interface in the unit, the volume fraction $i$ fluid will be a value between 0 and 1 . The volume fraction equation is given by:

$$
\frac{\partial\left(\rho \alpha_{i}\right)}{\partial t}+\frac{\partial\left(\rho u \alpha_{i}\right)}{\partial x}+\frac{\partial\left(\rho v \alpha_{i}\right)}{\partial y}+\frac{\partial\left(\rho w \alpha_{i}\right)}{\partial z}=0
$$

Where $\alpha_{\mathrm{i}}$ is the volume fraction of any individual fluid.

For the two-phase flow of cement slurry displacing drilling fluid, there exists:

$$
\begin{aligned}
& a_{1}+a_{2}=1 \\
& \rho=a_{1} \rho_{1}+a_{2} \rho_{2}
\end{aligned}
$$

Where $\rho_{1}$ and $\rho_{2}$ represent the density of cement slurry and drilling fluid, respectively. $\alpha_{1}$ and $\alpha_{2}$ represent the volume fraction of slurry and drilling fluid in a control body, respectively.

\subsection{Solutions and Boundary Conditions}

The analysis of fluid dynamics in the annular region is based on two-phase flow simulation in which one fluid dis- places another with distinct physical properties. The solution of the equations of motion for the problem provides the evolution of the shape of the interface between two fluids along their path. Computational Fluid Dynamics is a powerful tool for describing such processes.

The general idea of numerical simulation is as follows: through discretizing the continuous and analytical flow field into a number of nodes in simulation, the density, velocity and pressure changing with time on each node can be calculated. Moreover, the position of the leading and following edges in the displacement interface can be tracked in realtime if building two assumption surfaces .

The methods used to discretize the differential equations are as follows: using single-precision finite volume method for space discretization, using implicit scheme for time discretization, using the first-order up-wind finite volume method for the convective term discretization, and using the SIMPLE algorithm to solve the coupling of pressure and velocity fields.

\subsubsection{Discretization of Momentum Conservation Equations and Continuity Equation}

The finite volume method is used to discretize the momentum conservation and continuity equation. The basic idea is as follows: discretizing the solution area into many finite size grids; constructing a control body that includes at least one grid node and the body's volume is $V$; integrating the momentum conservation equations and continuity equation in the control body.

$\int_{V} \frac{\partial(\rho \varphi)}{\partial t} d V+\int_{V} \operatorname{div}(\rho \varphi \boldsymbol{U}) d V=\int_{V} \operatorname{div}(\mu \operatorname{grad} \varphi) d V+\int_{V} F_{\varphi} d V$

The volume integral of divergence in the above equation can be translated into the surface integral by Gauss theorem. The discrete equation in $\Delta t$ time is then expressed as:

$\int_{\Delta t} \frac{\partial}{\partial t}\left(\int_{V} \rho \varphi d V\right)+\int_{\Delta t} \int_{A} \boldsymbol{n} \cdot \rho \varphi \boldsymbol{U} d A d t=\int_{\Delta t} \int_{A} \boldsymbol{n} \cdot(\mu \operatorname{grad} \varphi) d A d t+\int_{\Delta t} \int_{V} F_{\varphi} d V$

\subsubsection{Discretization of Volume Fraction Equation}

The discretized form using the first-order up-wind finite volume method for the volume fraction equation is as follows:

$\frac{\rho\left(\alpha_{i j}^{n+1}-\alpha_{i j}^{n}\right)}{\tau}+u \frac{\rho\left(\alpha_{i j}^{n}-\alpha_{i j-1}^{n}\right)}{h}+v \frac{\rho\left(\alpha_{i j}^{n}-\alpha_{i j-1}^{n}\right)}{h}+w \frac{\rho\left(\alpha_{i j}^{n}-\alpha_{i j-1}^{n}\right)}{h}=0$

Where $\tau$ and $h$ represent the time step and the length step of discrete grids respectively.

\subsubsection{Calculation of the Coupling of Pressure Field and Velocity Field}

The SIMPLE algorithm is used when calculating the coupling of pressure field and velocity field. The basic idea of the SIMPLE algorithm is as follows: 1) Calculation of the initial velocity field. The velocity can be obtained by using given pressure, which may be a group of hypothetical values or calculated from the last iteration, into the discrete momentum conservation equations. Unfortunately, the obtained velocity field does not always meet the continuity equation because of the inaccurate pressure values. Therefore, given 
pressure values need to be modified. The modification principle is as follows: the renewed velocity corresponding to the modified pressure can meet the continuity equation. 2) Calculation of the modified velocity field. Firstly, by plugging the relation between pressure and velocity derived from the discrete momentum conservation equations into the discrete continuity equation, the pressure correction equation can be obtained. The modified pressure can then be calculated, based on the pressure correction equation. Finally, the renewed velocity is obtained based on the modified pressure. Of course, checking the convergence of the renewed velocity is needed. If not, a next calculation of the velocity field will be started until the convergent velocity is obtained.

\subsubsection{Boundary Conditions}

The adopted boundary conditions in numerical simulation are as follows:

Velocity boundary condition should be met at the entrance and the inlet velocity is $1.0 \mathrm{~m} / \mathrm{s}$.

Laminar flow displacement should be met.

Outflow boundary condition should be met at the exit.

No slip at the walls should be allowed.

The time step is $0.01 \mathrm{~s}$.

\section{NUMERICAL SIMULATION MODEL}

\subsection{Fluids Constitutive Equations}

The choice of an adequate rheological model can also help in characterizing the fluid behavior. A model, such as the Herschel-Bulkey, combines both the viscoplastic behavior (as provided by the Bingham model) with the nonlinear shear thinning effects (as proposed by the power law model). Because the Herschel-Bulkey model is more robust to describe the relation between shear stress and shear rate, it is adopted here to describe the fluids (cement slurry and drilling mud) behavior in numerical simulation. The HerschelBulkey model can be expressed as:

$$
\tau=\tau_{0}+K \cdot \gamma^{n}
$$

Where $\tau_{0}$ is the yield point of the fluid, $K$ is the consistency index, $n$ is the shear thinning index, $\tau$ is the shear rate. Equation 11 can be alternatively represented as:

$$
\tau=\tau_{0}+\left(\mu_{\infty} \gamma\right)^{n}
$$

Where $\mu_{\infty}$ is related to $\mathrm{K}$ as, $\mu_{\infty}=K^{1 / n}$ with units of viscosity.

\subsection{Viscosity Ranking}

Maintaining viscosity ranking and density differences among cementing fluids in the annulus is a well-advocated best practice in oilfield cementing. Researchers have previously discussed the negative effects of displacing thick drilling fluids with relatively thin cement slurries by means of the Fluent software and field validation $[15,16]$. As fluid velocity and viscosity profiles change with respect to the radical distance from the axis of the wellbore, a parametric approach has been considered to study the effects of viscosity ranking. The concept of averaging local shear rates over the annular volume previously introduced by Sairam et al. [17] is used here.

$$
\gamma_{A v g}=C \times \frac{Q}{A \times\left(D_{o}-D_{i}\right)}
$$

Where $\gamma_{\text {Avg }}$ is called volume averaged shear rate (VASR), $C$ is the shear-rate coefficient, $A$ is the annular area, $Q$ is the volumetric flow rate, and $D_{\mathrm{o}}$ and $D_{\mathrm{i}}$ are the outer and inner diameters of the annulus, respectively.

Based on VASR, an apparent viscosity is calculated as below:

$$
\mu_{A p p}=\frac{\tau_{o}+\left(\mu_{\infty} \gamma_{A c g}\right)^{n}}{\gamma_{A c g}}
$$

\subsection{Physical Model of Numerical Simulation}

Assuming that casing eccentricity is only downward, the physical model of eccentric annulus in a horizontal well which is shown in Fig. (1) is built in the Fluent software. Because the geometrical shape of a physical model is regular and each boundary is simple, structured Hexahedral mesh diving operation is conducted for the physical model in order to increase the calculation velocity and accuracy. Table $\mathbf{1}$ describes the simulation geometries for the physical model.

Table 1. Simulaiton Geometries for the Physical Model

\begin{tabular}{|c|c|c|c|c|}
\hline Type & Diameter $(\mathbf{m m})$ & Model Length $(\mathbf{m m})$ & Inclination $\left({ }^{\circ}\right)$ & Casing Eccentricity \\
\hline \hline Wellbore & 230 & 20000 & 90 & $0 \sim 0.5$ \\
\hline Casing & 139.7 & & 0.5 \\
\hline
\end{tabular}

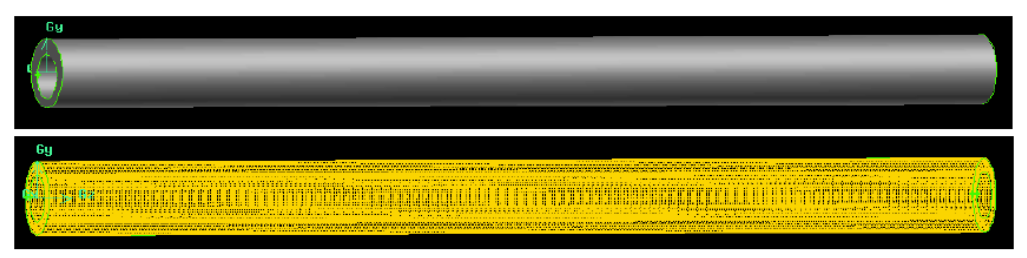

Fig. (1). Configuration of eccentric annulus and its mesh diving. 


\subsection{Fluid Performance Parameters}

Maintaining the performance parameters of drilling fluid unchanged, we change the slurry's and divide parameters into four cases for numerical simulation. In order to analyze the effects of density difference on the interface length and find the optimal density difference (defined as $\Delta \rho=\rho_{2}-\rho_{1}$ ) that makes the interface length minimum, we only change the slurry density in Case 1. Considering the effects of rheological parameters of the slurry on the optimal density difference, we change the shear thinning index, consistency index, and yield point of the slurry in Case 2, Case 3 and Case 4, respectively. Different fluid performance parameters are presented in (Table $\mathbf{2}$ to $\mathbf{5}$ ).

\section{RESULTS AND DISCUSSIONS}

\subsection{The Effects of Slurry Density on the Interface Length}

By taking the advantage of symmetry for the partial simulation in the Fluent software, the displacement interface shape can be extracted at 10 seconds under different $e$ and $\Delta \rho$, as shown in Figs. (2) through (7).

Based on the results presented in Figs. (2) through (7), we conclude that:

(1) Zero eccentricity means the casing is in the center of the wellbore. There is no difference in resistance for either the wide or narrow clearance. The buoyancy effects caused by density difference play a leading role here. Greater density difference can elongate the displace interface, which leads to severe slurry fingering in the annular lower side. Therefore, smaller density difference will achieve good displacement quality.

(2) When the casing is eccentric, where the slurry fingering happens depends on the relative relation of buoyancy and resistance. Slurry tends to advance in the annular upper side under smaller density difference because the buoyancy effects cannot overcome the resistance effects. Otherwise, the opposite results can be obtained under greater density difference.

Table 2. Performance Parameters of the Fluids in Case 1

\begin{tabular}{|c|c|c|c|c|c|}
\hline Fluid & $\boldsymbol{n}$ & $\boldsymbol{k}$ (Pa.s) & $\boldsymbol{\tau}_{\mathbf{0}}(\mathbf{P a})$ & $\boldsymbol{\rho}\left(\mathbf{g} / \mathbf{c m}^{\mathbf{3}}\right)$ & $\boldsymbol{\Delta} \boldsymbol{\rho ( \mathrm { g } / \mathbf { c m } ^ { \mathbf { 3 } } )}$ \\
\hline \hline Drilling fluid & 0.87 & 0.24 & 4 & 1.3 & $0.1 \sim 0.9$ \\
\hline Slurry A & 0.55 & 0.60 & 8 & $1.4 \sim 2.2$ \\
\hline
\end{tabular}

Table 3. Performance Parameters of the Fluids in Case 2

\begin{tabular}{|c|c|c|c|c|c|}
\hline Fluid & $\boldsymbol{n}$ & $\boldsymbol{k}(\mathbf{P a} . \mathbf{s})$ & $\boldsymbol{\tau}_{\mathbf{0}}(\mathbf{P a})$ & $\boldsymbol{\rho}\left(\mathbf{g} / \mathbf{c m}^{3}\right)$ & \multicolumn{1}{|c|}{1.3} \\
\hline \hline Drilling fluid & 0.87 & 0.24 & 4 & \multirow{2}{*}{$1.4 \sim 2.2$} \\
\hline Slurry B & 0.40 & 0.60 & 8 & $0.1 \sim 0.9$ \\
\hline Slurry A & 0.55 & 0.60 & 8 & 8 \\
\hline Slurry C & 0.70 & 0.60 & & \\
\hline
\end{tabular}

Table 4. Performance Parameters of the Fluids in Case 3

\begin{tabular}{|c|c|c|c|c|c|}
\hline Fluid & $n$ & $k$ (Pa.s) & $\tau_{0}(\mathrm{~Pa})$ & $\rho\left(\mathrm{g} / \mathrm{cm}^{3}\right)$ & $\Delta \rho\left(\mathrm{g} / \mathrm{cm}^{3}\right)$ \\
\hline Drilling fluid & 0.87 & 0.24 & 4 & 1.3 & \multirow{3}{*}{$0.1 \sim 0.9$} \\
\hline Slurry A & 0.55 & 0.60 & 8 & \multirow[t]{2}{*}{$1.4 \sim 2.2$} & \\
\hline Slurry E & 0.55 & 0.80 & 8 & & \\
\hline
\end{tabular}

Table 5. Performance Parameters of the Fluids in Case 4

\begin{tabular}{|c|c|c|c|c|c|}
\hline Fluid & $n$ & $k($ Pa.s) & $\tau_{0}(\mathrm{~Pa})$ & $\rho\left(\mathrm{g} / \mathrm{cm}^{3}\right)$ & $\Delta \rho\left(\mathrm{g} / \mathrm{cm}^{3}\right)$ \\
\hline Drilling fluid & 0.87 & 0.24 & 4 & 1.3 & \multirow{3}{*}{$0.1 \sim 0.9$} \\
\hline Slurry A & 0.55 & 0.60 & 8 & \multirow[t]{2}{*}{$1.4 \sim 2.2$} & \\
\hline Slurry G & 0.55 & 0.60 & 10 & & \\
\hline
\end{tabular}




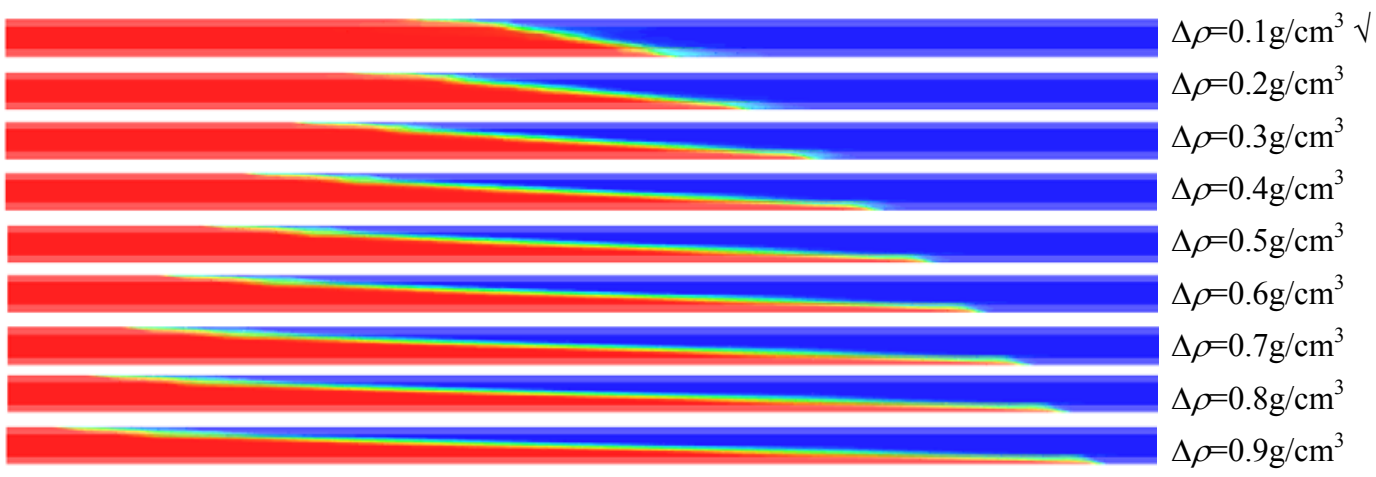

Fig. (2). Displacement interface shape for an eccentricity of 0 with different $\Delta \rho$.

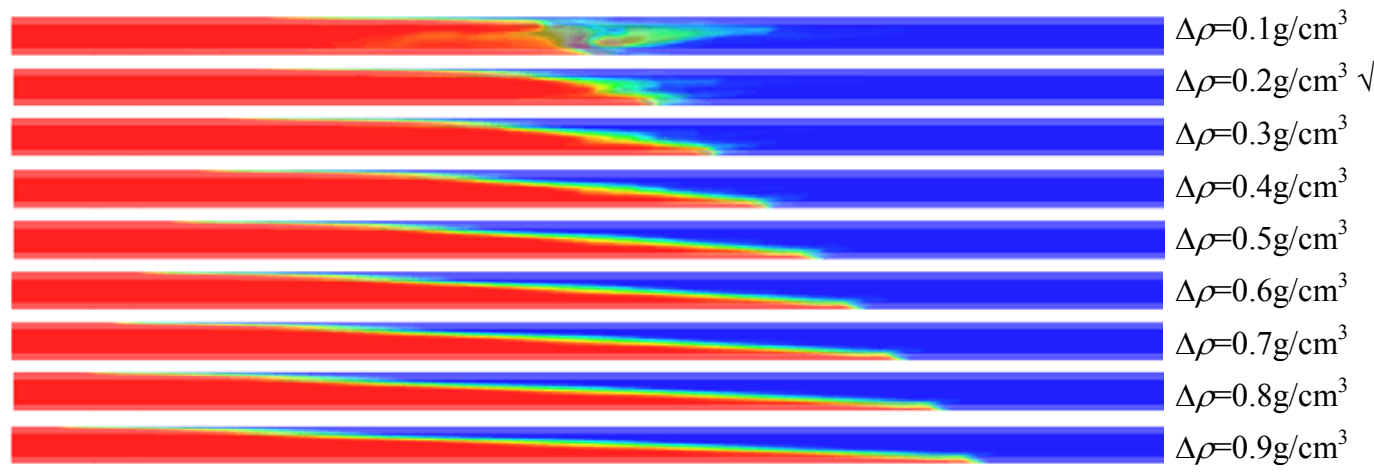

Fig. (3). Displacement interface shape for an eccentricity of 0.1 with different $\Delta \rho$.

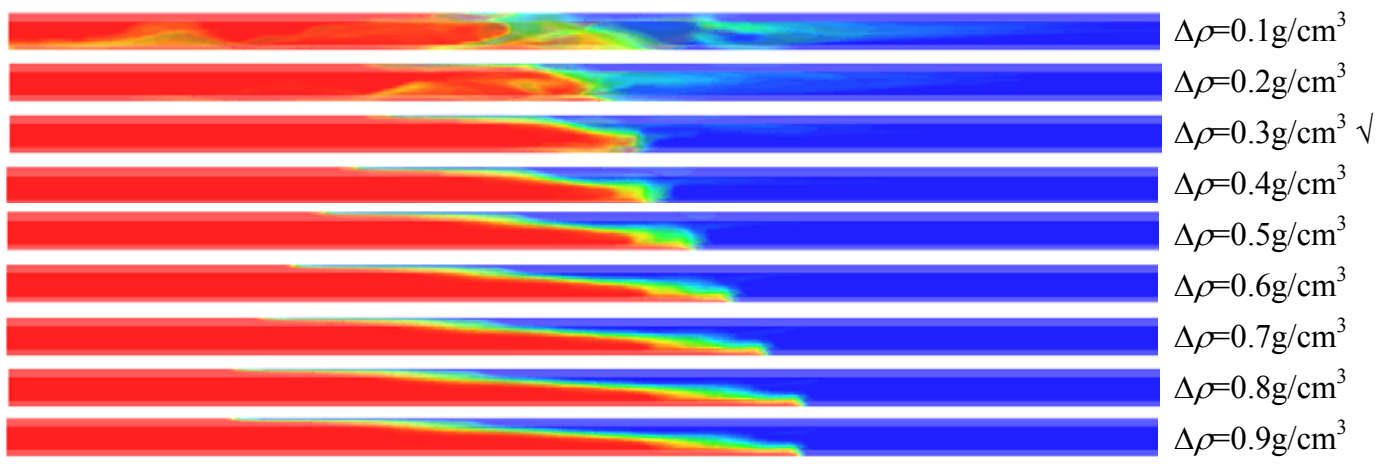

Fig. (4). Displacement interface shape for an eccentricity of 0.2 with different $\Delta \rho$.

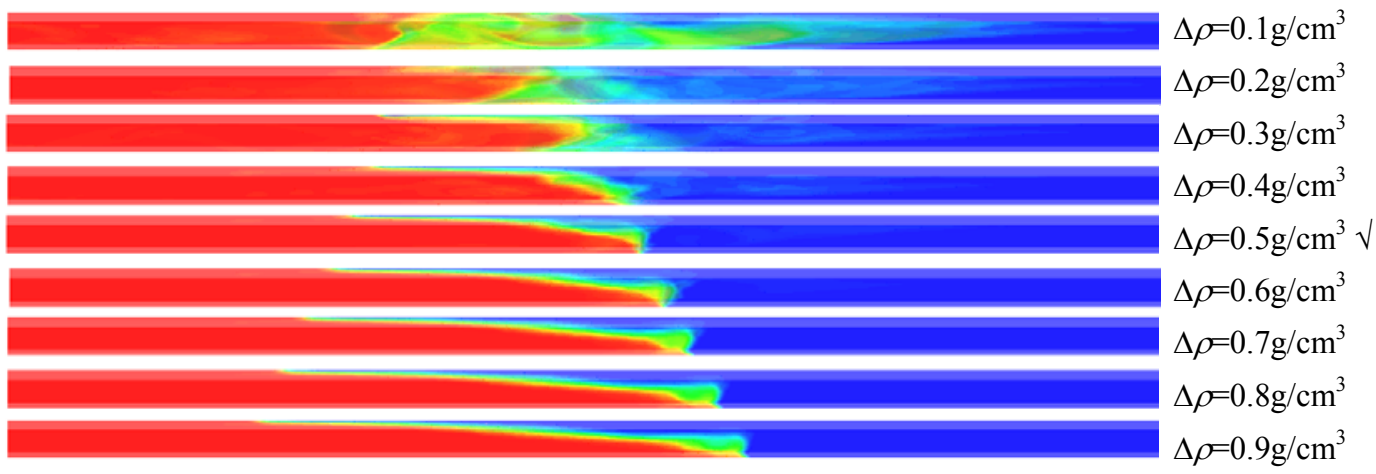

Fig. (5). Displacement interface shape for an eccentricity of 0.3 with different $\Delta \rho$. 


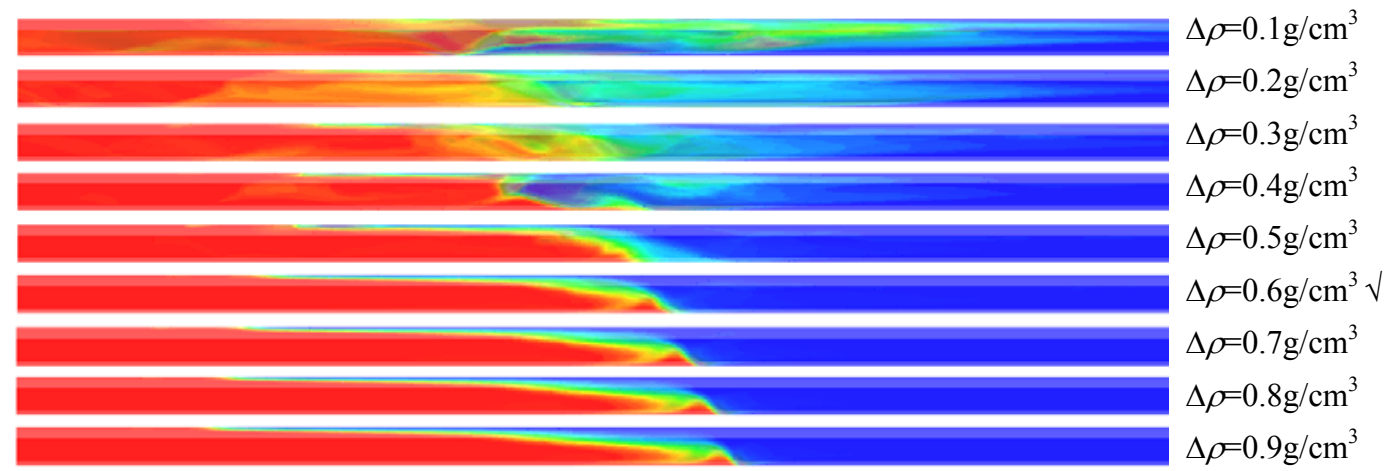

Fig. (6). Displacement interface shape for an eccentricity of 0.4 with different $\Delta \rho$.

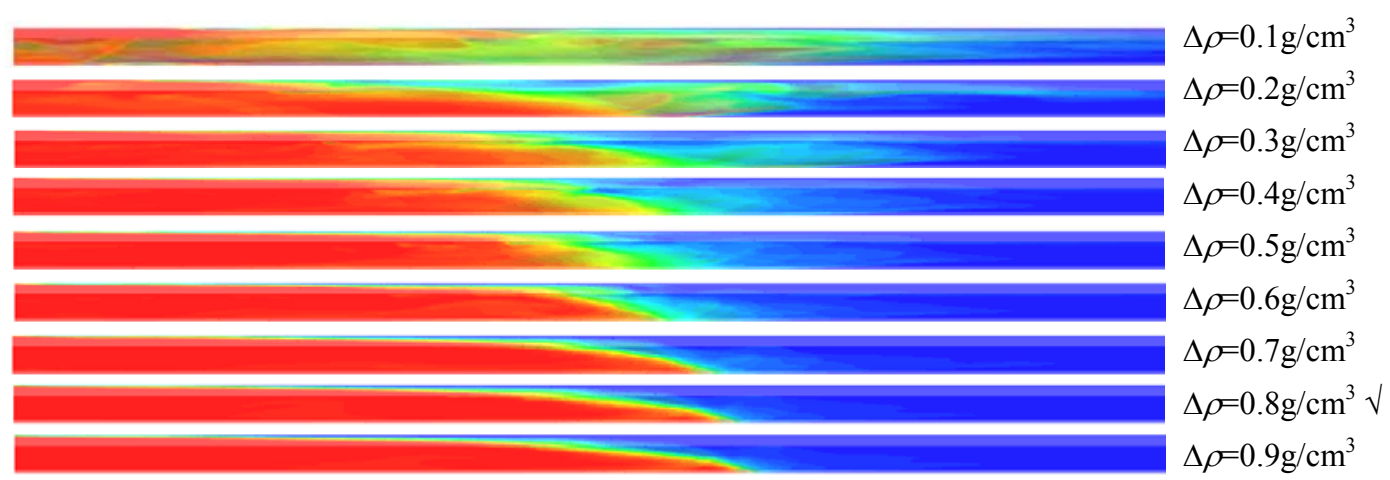

Fig. (7). Displacement interface shape for an eccentricity of 0.5 with different $\Delta \rho$.

(3) Comparison of the interface length under different $\Delta \rho$, it can be obviously seen that there exists an optimal density difference, under which the interface length is minimum because of the buoyancy balancing out the resistance. Fig. (8) illustrates the displacement interface length changing with density differences for a given eccentricity value. As shown in (Fig. 8), the optimized density differences corresponding to the eccentricity of $0.1,0.2,0.3,0.4$ and 0.5 are $0.2 \mathrm{~g} / \mathrm{cm}^{3}, 0.3 \mathrm{~g} / \mathrm{cm}^{3}, 0.5$ $\mathrm{g} / \mathrm{cm}^{3}, 0.6 \mathrm{~g} / \mathrm{cm}^{3}$ and $0.8 \mathrm{~g} / \mathrm{cm}^{3}$, respectively. Besides, the results are similar to Muhammad Zulqarnain's [9] obtained with CFD. Muhammad Zulqarnain's results are: 1) For a certain casing's eccentricity ( $e=0.15,0.3 、 0.6$ ) , slurry advances on the annular upper side with no density difference between slurry and spacer(spacer's density is equal to the drilling fluid's).However, slurry advancing happens on the annular low side when there exists density difference and the advancing extent is more severe. 2) For a certain density difference, when casing's eccentricity is 0.15 , 0.3 and 0.6 , the volume fraction of slurry is $82 \%, 85 \%$ and $84 \%$ respectively. So displacement effect is the best under casing's eccentricity 0.3 . We can conclude that there exist a reasonable matching relationship between density difference and casing's eccentricity.

\subsection{The Effects of Slurry's Rheological Parameters on the Optimal Density Difference}

In order to analyze the effects of rheological parameters of the slurry on the optimal density difference, we simulate several groups of interface shapes under the conditions with an eccentricity of 0.3 , density difference of 0.6 and various rheological parameters. By taking the advantage of symmetry for the partial simulation in the Fluent software, the displacement interface shape can be extracted at 10 seconds under different $n, K$ and $\tau_{0}$ as shown in Figs. (9) through (11).

Furthermore, the optimal density difference under various rheological parameters is given in the following plots:

Figs. (9) through (11) illustrate that the interface length is changing obviously with the shear thinning index, consistency index, and yield point of the slurry. We can conclude that the displacement effects can be improved by appropriately adjusting the rheological parameters.

Figs. (12) through (14) illustrate that the optimal density difference corresponding to the casing eccentricity will increase along with the increase of the shear thinning index, consistency index, and yield point of the slurry. We conclude that the optimal density difference which makes the interface length minimum is affected by both the casing eccentricity and the rheological parameters of the slurry. Furthermore, there exists a reasonable matching relationship among them. In order to minimize the intermixing extent of two-phase fluids and slurry fingering effects in cementing operations, it is necessary to take the comprehensive influence of casing eccentricity, slurry density, and rheological parameters into consideration to design slurry parameters properly. Finally, this study demonstrated that higher displacement efficiency and better displacement quality were obtained. 


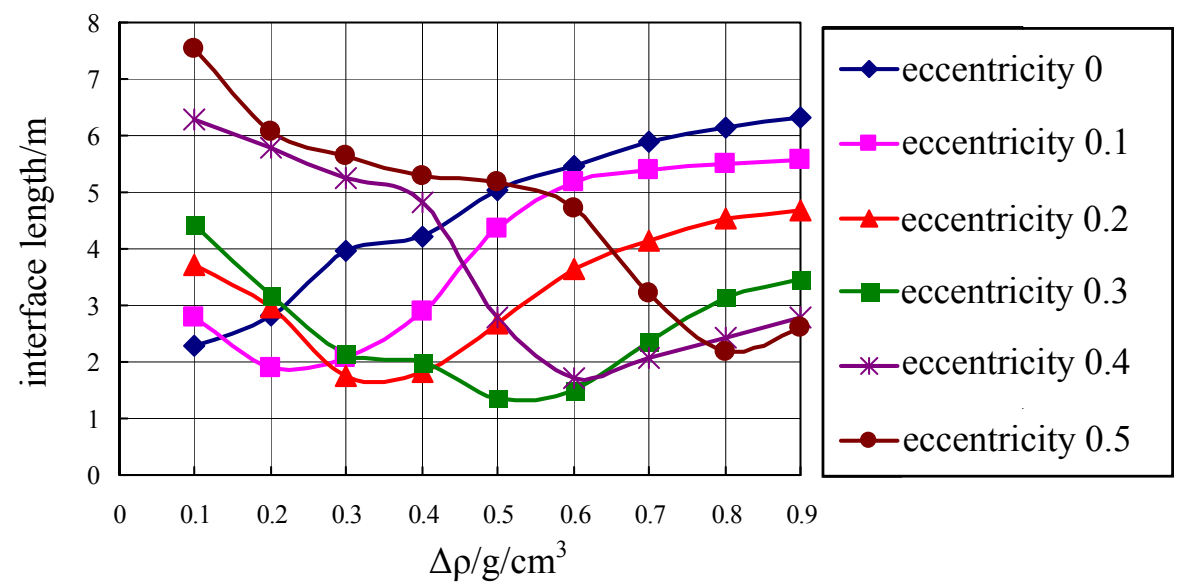

Fig. (8). Displacement interface length in different density difference.

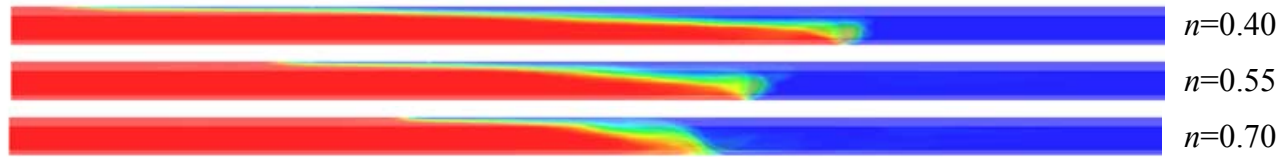

Fig. (9). Displacement interface shape for $e 0.5$ and $\Delta \rho 0.6$ with different.

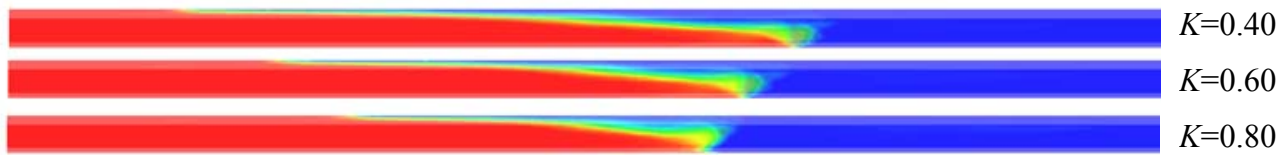

Fig. (10). Displacement interface shape for $e 0.5$ and $\Delta \rho 0.6$ with different $K$.

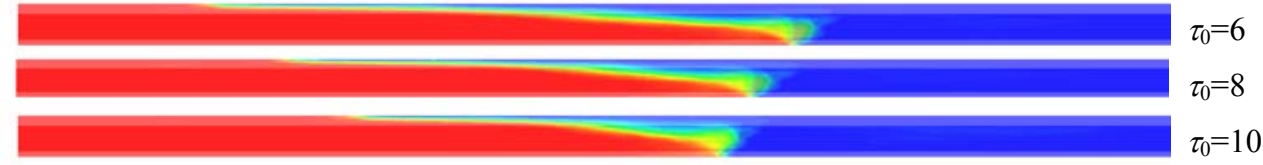

Fig. (11). Displacement interface shape for $e 0.5$ and $\Delta \rho 0.6$ with different $\tau_{0 \text {. }}$

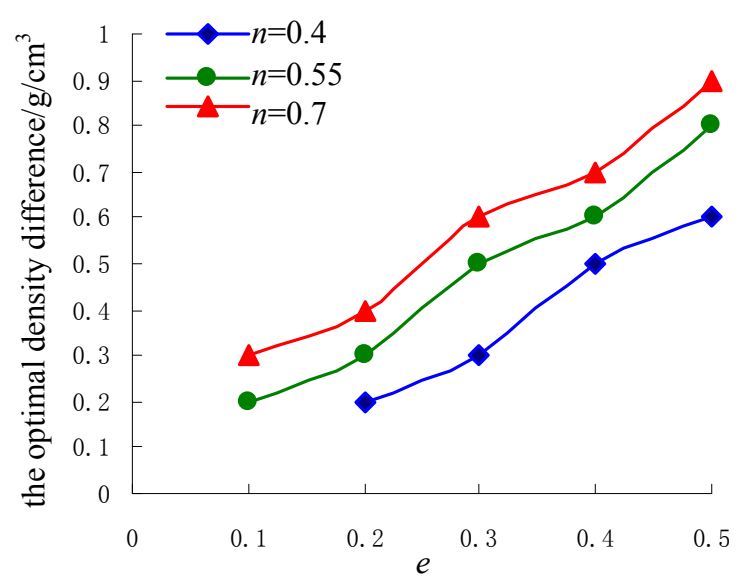

Fig. (12). The optimal density difference with $e$ under different $n$.

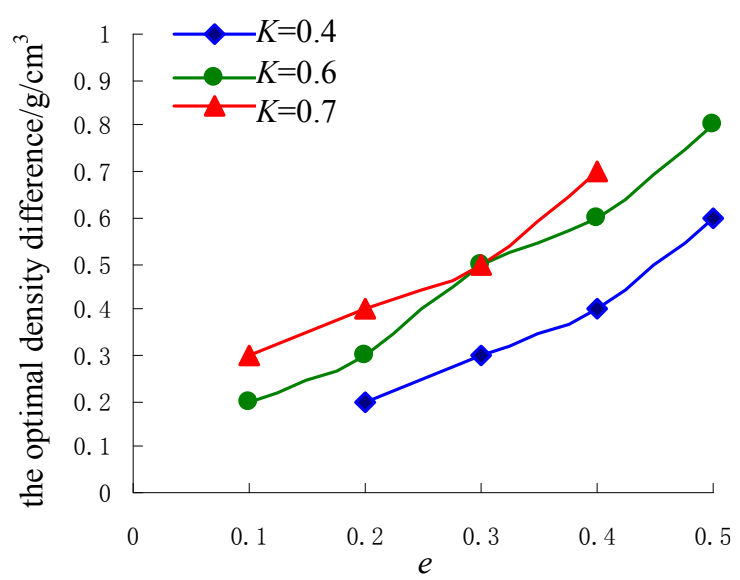

Fig. (13). The optimal density difference with $e$ under different $K$. 


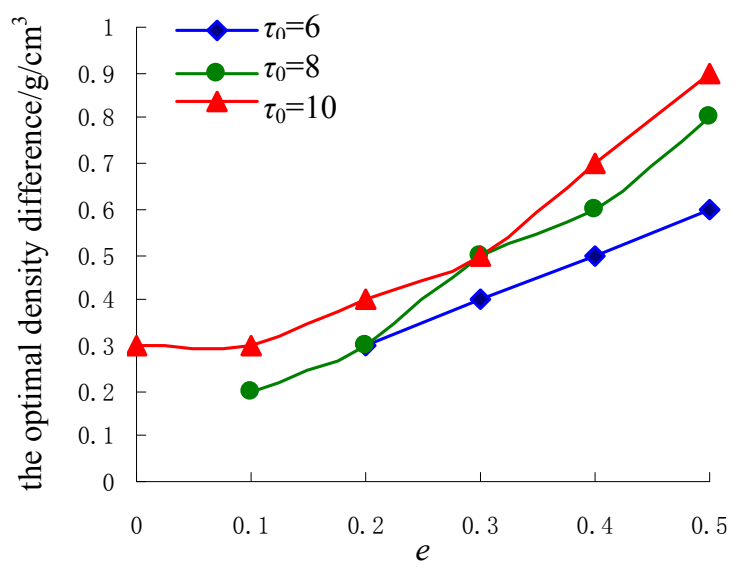

Fig. (14). The optimal density difference with $e$ under different $\tau_{0}$.

\section{CONCLUSIONS}

(1) Shorter interface and better displacement quality can be obtained by means of smaller density differences when the casings are centralized in horizontal wells.

(2) Both larger and smaller density difference can adversely affect the displacement quality, when the casings are eccentric. There exists a range of density differences for achieving the most stable displacement interface. If the density differences are smaller, the buoyancy effects cannot overcome the resistance effects. As a result, intermixing at two-phase fluids interface will become deteriorated and the slurry fingering in the wide clearance of the annulus will occur. If the density differences are greater, the buoyancy effects can exceed the resistance effects. Finally, slurry fingering in the narrow clearance of annulus will happen.

(3) The shear thinning index, consistency index and yield point of slurry have an obvious effects on the interface length. The optimal density difference corresponding to the casing eccentricity will increase along with the increase of shear thinning index, consistency index and yield point of the slurry. In order to minimize the intermixing extent of two-phase fluids and slurry fingering effects in cementing operations, it is necessary to take the comprehensive influence of casing eccentricity, slurry density, and rheological parameters into consideration to design slurry parameters properly. Finally, higher displacement efficiency and better displacement quality will be obtained.

\section{CONFLICT OF INTEREST}

The author(s) confirm that this article content has no conflicts of interest.

\section{ACKNOWLEDGEMENT}

The paper is supported by Natural Science Foundation of Heilongjiang Province of China (Project No. QC2012C021).

\section{REFERENCES}

[1] P. Sabot, and O. Hassager, "Displacement of one Newtonian fluid by another: density effects in axial annular flow", International Journal of Multiphase Flow, vol. 23, pp. 695-701, 1997.

[2] S. Pelipenko, and I.A. Frigaard, "Visco-plastic fluid displacements in near vertical narrow eccentric annuli: prediction of travellingwave solutions and interfacial in-stability", Journal of Fluid Mechanics, vol. 520, pp. 343-377, 2004.

[3] S. Pelipenko, and I.A. Frigaard, "Two-dimensional computational simulation of eccentric annular cementing displacements", IMA Journal of Applied Mathematics, vol. 69, pp. 557-583, 2004.

[4] E.S.S Dutra, A.L. Martins, and C.R. Miranda, "Dynamic of fluids substitution while drilling and completion long horizontal section well", at the SPE Latin American and Caribbean Petroleum Engineering Conference, 2005, pp. 1-8.

[5] J.B. Yang, J.M. Deng, Z.H. Li, and Y. Chen, "Effect of density difference on displacement efficiency during low-rate cementing", Petroleum Drilling Techniques, vol. 35, pp. 79-82, 2007.

[6] J.B. Yang, D. Jianmin, Y.Q. Feng, H.Y. Zhao, and D.D. Wang, "Numerical simulation of effects of density difference on displacement efficiency at low cement slurry velocity", Petroleum Drilling Techniques, vol. 36, pp. 62-65, 2008.

[7] S. Pks, and K.B. Yerubandi, "Slim-Well Completions: A 3D numerical approach for displacement to design effective cementing fluids", at the SPE Trinidad and Tobago Energy Resources Conference, 2010, pp. 1-9.

[8] P.E. Aranha, C.R. Miranda, and J.V.M. Magalhaes, "Dynamic aspects governing cement plug placement in deepwater wells", SPE Drilling \& Completion, vol. 26, pp. 341-350, 2011.

[9] M. Zulqarnain, "Simulations of the primary cement placement in annular geometries during well completion using computional fluid dynamics(CFD)", Msc Thesis, The Craft and Hawkins Department of Petroleum Engineering, Louisiana State Univ, USA, 2012.

[10] Y.G. Zheng, "Displacement mechanism of laminar flow cementing in deviated wells", Acta Petrolei Sin, vol. 16, pp. 133-138, 1996.

[11] Y.G. Zheng, D. Fang, and J.F. Hao, "A theoretical and experimental study of cementing for horizontal wells", Journal of Hydrodynamics, vol. 11, pp. 19-23, 1996.

[12] Z.M. Li, C.W Wang, and C.Q. Wang, "Numerical simulation of cement displacement in eccentric annulus at highly deviated wells", Petroleum Drilling Techniques, vol. 40, pp. 40-44, 2012.

[13] S.J. Zhang, L. X. and Z.M. Wang, "Numerical simulation studies on the influence law of density difference on cementing displacement interface in the horizontal well", Drilling \& Production Technology, vol. 35, pp. 15-17, 2012.

[14] S.J Zhang, L. X. and Z.M. Wang, "Cement displacement flow law affected by positive density difference in the eccentric annulus of the horizontal well", Science Technology and Engineering, vol. 13, pp. 1011-1015, 2013.

[15] R. M. Beirute, and R. W. Flumerfelt, "Mechanics of the displacement process of drilling muds by cement slurries using an accurate rhe-ological model", at the SPE Offshore Technology Conference, 1977, pp. 2-5.

[16] M. Savery, and R. Darbe, "Predicting mud removal during cementing-A new and simple approach", at the 2007 AADE National Technical Conference and Exhibition, 2007, pp. 2-7.

[17] P.K.S. Sairam, M. Savery, and R. Morgan, "Accurate and fast method for predicting actual top-of-cement depths in eccentric wellbores", at the SPE North Africa Technical Conference and Exhibition, 2010, pp. 3-8. 